\title{
Processing and Quality Evaluation of Additive Manufacturing Monolayer Specimens
}

\author{
Christiane Wendt, ${ }^{1,2}$ Severo Raúl Fernández-Vidal, ${ }^{1}$ Álvaro Gómez-Parra, ${ }^{1}$ \\ Moisés Batista, ${ }^{1}$ and Mariano Marcos ${ }^{1}$ \\ ${ }^{1}$ Mechanical Engineering and Industrial Design Department, University of Cadiz, Faculty of Engineering, \\ Avenida de la Universidad de Cadiz, No. 10, Puerto Real, 11519 Cadiz, Spain \\ ${ }^{2}$ Department of Automotive and Aeronautical Engineering, University of Applied Sciences Hamburg, \\ Faculty of Engineering and Computer Science, Berliner Tor 21, 20099 Hamburg, Germany
}

Correspondence should be addressed to Christiane Wendt; christiane.wendt@alum.uca.es

Received 19 January 2016; Revised 31 March 2016; Accepted 20 April 2016

Academic Editor: Ying Li

Copyright ( $) 2016$ Christiane Wendt et al. This is an open access article distributed under the Creative Commons Attribution License, which permits unrestricted use, distribution, and reproduction in any medium, provided the original work is properly cited.

\begin{abstract}
Although its importance has increased significantly, Additive Manufacturing is not yet a fully accepted industrial manufacturing process for load-carrying parts. The future success of the process group depends on its standardization. This work proposes a methodology for the design, manufacturing, and quality evaluation of specimens manufactured by Fused Layer Modeling that are composed of only one layer (so-called monolayers). The processing methodology and properties of monolayers have not been studied systematically yet. A first systematic design of monolayers for mechanical testing is presented. Rectangular and circular monolayers adapted to the loads of tensile and compression testing are manufactured using different trajectory strategies. Frequently occurring macro- and microgeometrical defects are evaluated and categorized in order to optimize the part quality. This work also studies the effect of some manufacturing parameters such as the gap between print head and machine bed, trajectory strategy, bed leveling, and temperatures on part quality. The most suitable specimens are tested mechanically in tensile or compression tests. In the case of study, tensile strength values are only $8.6 \%$ lower than the values for reference tests on the unextruded filament. However, the properties deviate more strongly for compression tests which may be due to the selected specimen geometry.
\end{abstract}

\section{Introduction}

Additive Manufacturing (AM) is gaining importance for the manufacturing not only of prototypes and tools, but also for serial load-carrying parts $[1,2]$. It is even starting to replace conventional manufacturing processes where complex parts with small lead-time and lot sizes are needed, more so if otherwise specialized tools would be needed $[3,4]$. While AM was invented more than twenty years ago under the term Rapid Prototyping [4], the market interest has only recently increased strongly with AM now producing about $29 \%$ of final parts [1].

AM technologies are manufacturing processes which create the physical part by adding material incrementally based on digital information. This information is provided via a three-dimensional CAD-model that is converted to a tessellated format and sliced virtually [5] (Figure 1).

This way of obtaining a part leads to new design possibilities and can therefore produce parts with a very high stiffnessto-weight-ratio [4].

Parts manufactured with track-based AM processes are generally anisotropic within and between layers [4, 6-8]. For Fused Layer Manufacturing (FLM), the single tracks and layers (monolayers) are similar to the fibers and layers that are part of laminated fiber-reinforced composite materials [9]. The binding between the tracks or fibers and between layers has a strong influence on the properties of the multilayer part both for fiber-reinforced composites and for parts manufactured using a track-based AM process (such as Electron Beam Melting, Fused Layer Manufacturing, or Stereolithography) 


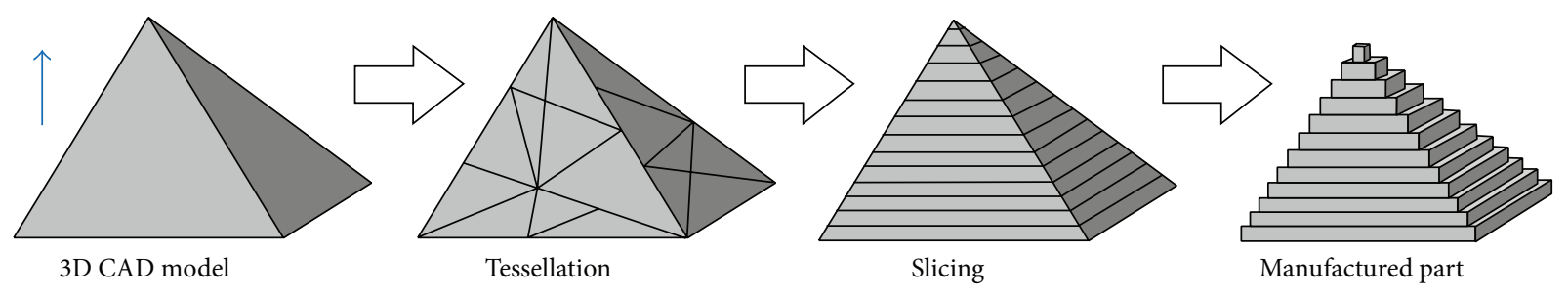

FIgURE 1: General AM process (blue = build direction).

[9]. For these processes, the in-plane mechanical behavior of a multilayer part can be approximated knowing the properties of the monolayers and their orientation within the part. Stiffness matrices and equations that are normally used for fiber-reinforced composites were applied to Fused Deposition Modeling ${ }^{\circledR}$ in a first successful approach in 2003 [9].

Still, AM technologies have not fully entered into industries that are highly sensible to safety factors, such as the aeronautical sector. Industry stakeholders link the future acceptance of AM technologies to the development of standards and norms regarding, among others, manufacturing processes and mechanical testing [10].

Based on these findings, this paper establishes the following state-of-the-art key points regarding standardization of the AM process group:

(i) For AM, no international standardized mechanical test procedures or specimen designs exist yet [11, 12]. Some standards are published or in draft by ISO and ASTM $[13,14]$, but they pertain neither to mechanical testing procedures of monolayers nor to specimen design.

(ii) Most of the mechanical tests conducted on FLM materials have been tensile tests so far, as can be found in $[15,16]$. A great majority of them use existing standards for thermoplastics or fiber-reinforced composites without further justification of the geometry and testing conditions (such as the test speed) nor of the test procedures. The test speed especially can have an influence on the strength values measured for each specimen. No complete methodology for design, manufacturing, and analysis of specimen properties was found for AM processes.

(iii) Conventional dumbbell specimen designs are unsuitable for FLM mechanical testing, as the nonparallel tracks may cause stress concentrations away from the specimens' gauge area [6]. This may be applicable to other road-based AM processes as well.

Because of this, it is necessary to establish a methodology that can be used for extracting relevant mechanical properties from simple mechanical tests like tensile tests. For these tests, new specimen designs, testing procedures, and so forth have to be defined, evaluated, and benchmarked against each other. Numerous benchmarks may lead to the proposal of new standard procedures that are a good approximation of the theoretical optimum for each combination of possible raw materials and AM processes.

As a first step in this large process, it seems best to start with developing a methodology for FLM monolayers. Monolayers are the biggest basic elements of road-based AM processes. Unlike single tracks, they also exist in technologies that use sheet-laminating or masks. This allows an adaptation of the methodology for any of the AM processes if needed. Also, for in-plane properties, the binding between layers has little influence on the multilayer. Therefore, monolayers can be used as a first reference for evaluating some bulk properties of multilayer parts [9]. While single roads are sometimes investigated [9], no efforts have been undertaken to systematically manufacture monolayers additively and their usefulness for mechanical tests is so far unknown. Moreover, it is necessary to establish a completely new methodology for the processing of AM monolayers and for performing a quality analysis.

This work is focused on advancing the development of AM specific design, manufacturing, and quality evaluation processes, starting with FLM monolayers. It proposes a methodology that can be followed for the whole iterative design, manufacturing, and testing cycle. This methodology is applied to compression and tensile tests of polylactic acid (PLA) specimens. The monolayers have cylindrical and rectangular geometries, respectively.

\section{Materials and Methods}

The methodology followed in this work is iterative (Figure 2). Starting with the parameters and geometry definition of suitable specimens, the samples are manufactured and evaluated regarding their quality. This evaluation includes micro- and macrogeometrical and mechanical aspects.

This stage is followed by a thorough analysis of the obtained data, including finding the optimum test speed for the selected AM process and material. The micro- and macrostructures of the specimens before and after mechanical testing are compared and the defects are related to the mechanical properties. During manufacturing or quality evaluation, the results may show that the selected parameters or design are unsuitable for the properties of the selected material or the specific manufacturing difficulties of the FLM process. In this case, the methodology includes the possibility of adapting the inputs and starting the development loop again even at intermediate stages.

The mechanical properties of the specimens depend on the orientation of the tracks within the FLM part. In this 


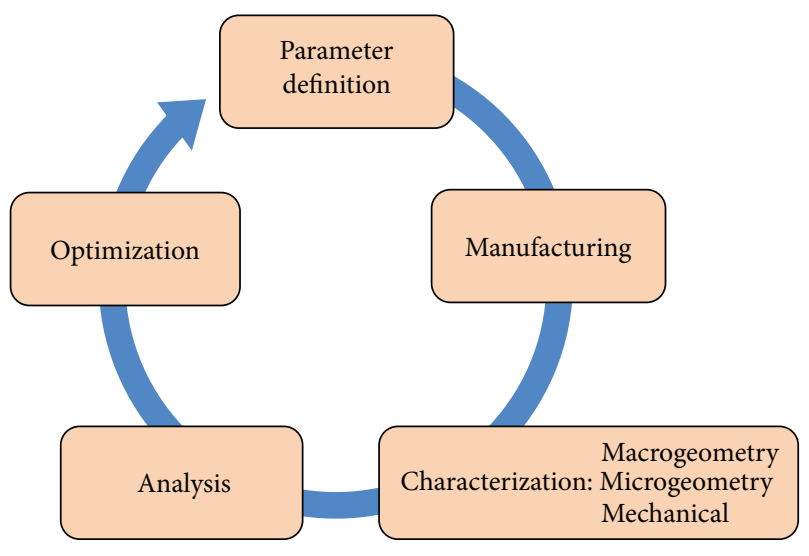

Figure 2: Central iterative methodology.

paper, the tracks are oriented in such a way that the maximum strength can be measured. For tensile testing, the tracks are parallel to the loading direction in the gauge area. This way, each track is stressed individually under the action of a unidirectional force. For compression testing, the tracks are oriented perpendicular to the loading direction to avoid the buckling of single tracks. The reduction of mechanical properties when orienting the tracks out-of-axis in tensile tests was already investigated and demonstrated by other authors for FLM [9]. By testing for maximum strength, the monolayers' properties can be compared to literature values for the raw material.

The selected material for this study is polylactic acid $\left(\mathrm{C}_{3} \mathrm{H}_{4} \mathrm{O}_{2}\right)$, also called PLA. It is a thermoplastic polymer that is made from renewable raw materials such as cornstarch or sugarcane and is therefore biodegradable. It is considered more ecologically friendly than, for example, ABS. PLA is commonly used as a raw material for FLM due to its physicochemical properties. PLA melts between $433 \mathrm{~K}$ and $463 \mathrm{~K}$ and is well usable at temperatures of up to $513 \mathrm{~K}$. At higher processing temperatures, the viscosity decreases [19].

The raw material has a density of $1.24 \mathrm{~g} \cdot \mathrm{cm}^{-3}$ that can decrease by up to $25 \%$ during the FLM process. The melt volume index of PLA is higher than $1 \mathrm{~cm}^{3} \cdot \mathrm{min}^{-1}$. An index higher than $0.7 \mathrm{~cm}^{3} \cdot \mathrm{min}^{-1}$ implies a good ease of flow for use in FLM [20].

PLA shows stress whitening (also called crazing) when subjected to tensile loads and is relatively ductile, which allows easy analysis of the tensile tests with videos and microscopy. The PLA is supplied on a spool with a filament diameter of $1.7 \mathrm{~mm}$. A Shimadzu AG-X series universal testing machine is used for mechanical testing. It has a maximum load cell capacity of $50 \mathrm{kN}$ and a displacement resolution of $0.0208 \mu \mathrm{m}$.

A series of reference tensile tests are performed on the unextruded filament with a test speed of $1 \mathrm{~mm} / \mathrm{min}$ (equivalent strain rate about $0.00021 \mathrm{~s}^{-1}$ ) and under otherwise identical testing conditions as in the tensile tests performed on the FLM monolayers; see below. The tensile properties for this test speed are converted to equivalent values corresponding to the optimum test speed of the monolayers, allowing easier comparison.
2.1. Parameter Definition and Manufacturing. The FLM monolayers are tested in tensile and compression tests. The simplest geometry types suitable to the applied loads are selected for this purpose. They are flat rectangles for tensile testing and hollow cylindrical tubes for compression testing. Their optimum dimensions depend not only on the load path, but also on the manufacturing process, additive fabricator, and material.

The material is processed on the additive fabricator CubeX ${ }^{\mathrm{TM}}$ by $3 \mathrm{D}$ Systems Inc., depositing it in tracks according to predefined trajectory strategies; see Figure 3. For compression specimens, helicoidal trajectories are chosen. One print head with an opening diameter of $0.75 \mathrm{~mm}$ is used.

2.2. Micro- and Macrogeometrical Characterization. According to [21], the mechanical properties of structures are scaleinvariant if these structures are bigger than their smallest basic element. This means that, for FLM parts composed of multiple tracks longer than their diameter, the absolute dimensions of the part do not significantly influence the properties and the mechanical behavior of the part. Only the ratios between the main dimensions have to be investigated. Additionally, the effect of other design and manufacturing parameters on the suitability of AM monolayers for mechanical testing is evaluated, in some cases qualitatively. These parameters are extrusion temperature, trajectory strategy, starting point location, bed leveling, and z-gap.

In order to be able to judge the suitability of the monolayers for mechanical testing, some quality criteria are defined:

(i) Defects should be representative of a multilayer part and not impede the structural integrity. A low number and size of defects in microgeometry, low porosity, and a lack of notable macrostructure defects contribute to this.

(ii) Form and dimensions shall be according to the specifications and may not be permitted to impede the gripping or placement of the specimens in the universal testing machine. This means good dimensional accuracy, low form deviations, very low out-of-plane deformations, and a uniform layer thickness.

(iii) Mechanical behavior shall be such that the measurement and statistical treatment of the modulus and strength data are possible.

The specimens that have the highest quality according to these criteria are selected for systematic mechanical testing. As no standards or preliminary work exist yet, the optimum test speed is determined by analyzing the resulting mechanical properties for various test speeds. This analysis takes into account that the optimum test speeds for determining the strength and modulus are often nonidentical (e.g., ISO 604).

2.3. Tensile Testing. Tensile tests are performed at the four traction speeds: $1,1.25,2$, and $2.5 \mathrm{~mm} / \mathrm{min}$. They are within the range of test speeds found in standards for mechanical testing of thermoplastic materials or fiber-reinforced thermoplastics. These two materials are similar to FLM multilayers. 


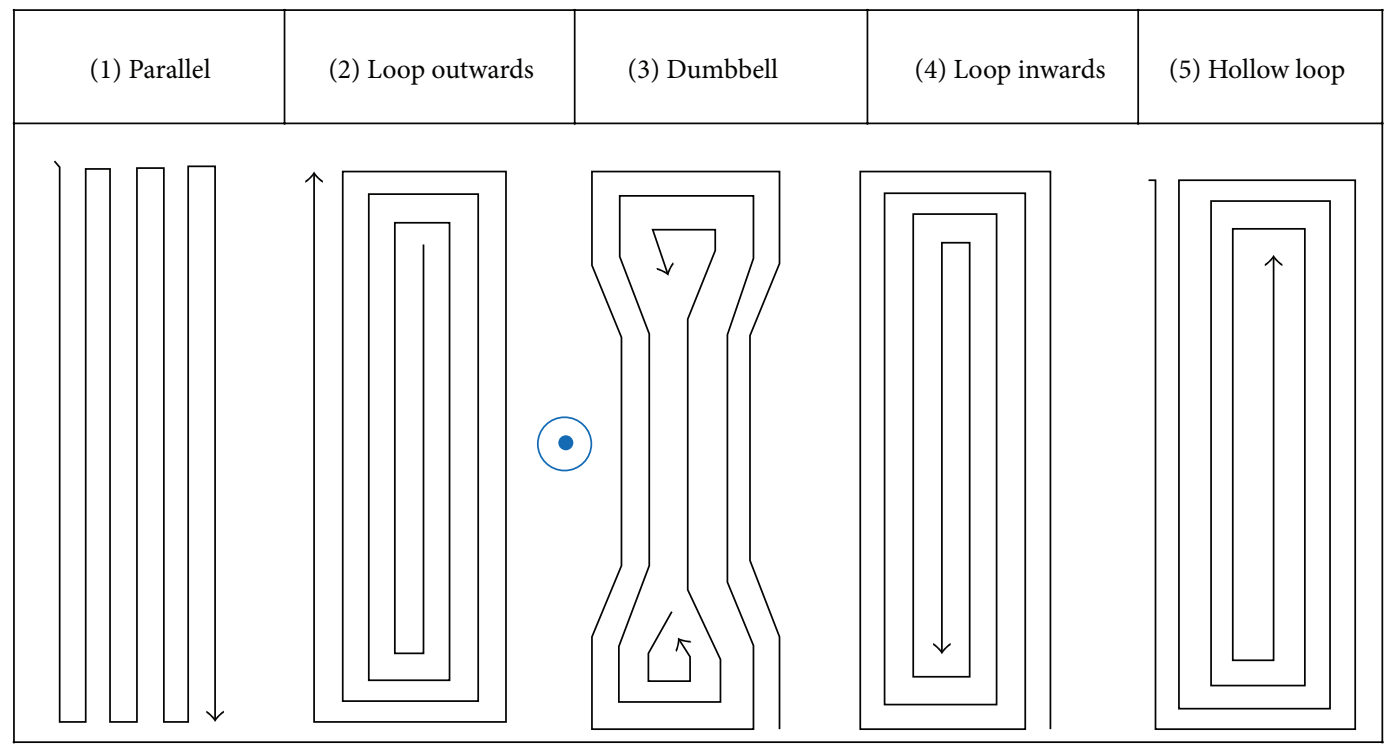

FIGURE 3: Trajectory strategies for tensile specimens (blue = build direction).

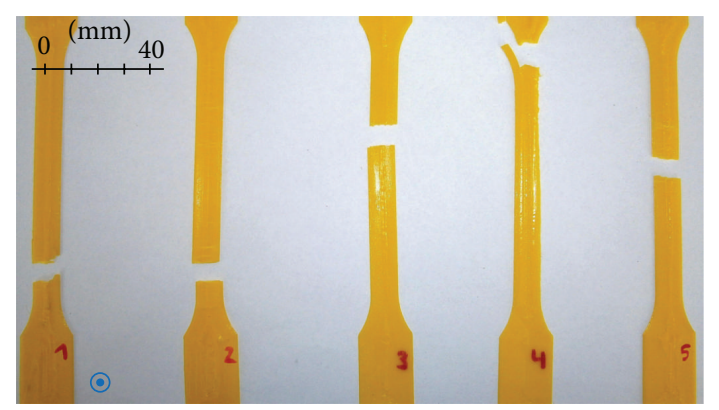

FIGURE 4: Fracture location on FLM dumbbell specimens (blue = build direction).

The optimum test speed may be different for each material and manufacturing process. It is the extreme value in the strength or modulus results obtained at different test speeds.

For tensile testing, file-teeth grips fix the specimens in position with a gauge length of $80 \mathrm{~mm}$. The file-teeth avoid slipping while not damaging the grip area of the specimens critically. At least seven nominally identical specimens were manufactured and are tested for each test speed, allowing a statistical treatment of the results. For microgeometrical analysis, roughness tester Mahr Perthometer Concept is used. It is equipped with a PGK 120 drive unit and MFW250 probes. Optical and scanning electron microscopy are used for full analysis of the sample geometry and defects, complemented by video recordings of the mechanical tests.

Additionally, qualitative tensile tests on conventional dumbbell specimens serve to confirm or negate the tendencies found by Ahn et al. [6]. The ISO 3167 type A specimens fracture within the grip area in one out of five cases, validating the prior findings; see Figure 4.

2.4. Compression Testing. The same geometry characterization procedure is applied to compression specimens. Based on existing standards for thermoplastic materials and the results of the compression tests, the possible optimum test speeds 1,2 , and $5 \mathrm{~mm} / \mathrm{min}$ are tested. An additional higher or lower test speed would be selected according to the data if an extremum of the properties did not exist within this range of test speeds. The specimens are compressed between two fixed platens with a diameter of $100 \mathrm{~mm}$ and an initial distance of about $20 \mathrm{~mm}$ depending on the exact specimen height.

\section{Results and Discussion}

3.1. Parameter Effects on Macro- and Microgeometry. As some manufacturing defects occur frequently and their type and gravity are often varied, a catalogue of the most frequent defects is created; see Figure 5.

In the catalogue, the defects are sorted by their spatial extent (zero- to three-dimensional), meaning that it can be expanded for all other AM processes:

(i) 0D: these defects are the least critical and negligible if they occur in low density and size. They are roughly point-shaped.

(ii) 1D: these defects are typically concentrated to only one track.

(iii) 2D: all in-plane deformations and defects are included.

(iv) 3D: this category contains out-of-plane deformations and global defects.

The dimensional accuracy of the produced specimens is sometimes relatively low, especially for the thickness of the flat specimens and the diameter of the circular tubes. As the variance of the deviations is low, the accuracy could be improved by adjusting the process or the design parameters to compensate the suspected systematic errors. The length of 


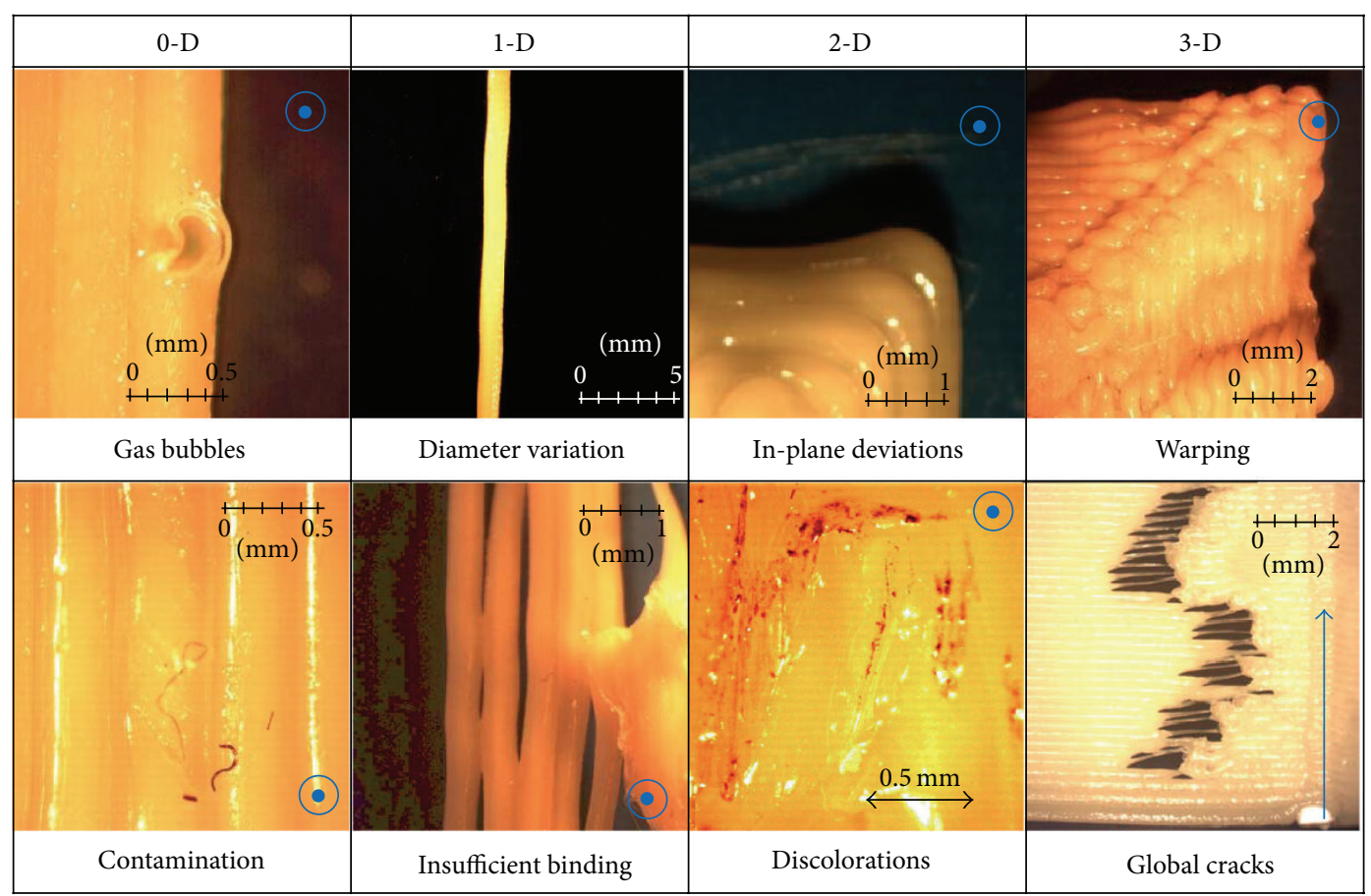

FIGURE 5: Classification examples for FLM monolayer defects (blue: build direction).

the tensile specimens and the wall thickness of the compression specimens have the highest dimensional accuracy. The differences in the location of the best and worst accuracy are most likely due to the nature of the specimens' geometries that influence thermal phenomena.

Five different trajectory strategies are defined for tensile specimens; see Figure 3. The hollow center of design number 5 compensates the extrusion of some excess material that occurs for designs 1-4. For compression specimens, only one trajectory strategy can be manufactured with the software and hardware at hand: the material is deposited in loops, causing a slight material accumulation at the routes that connect the layers. Helicoidal trajectories would be more suitable than the chosen fixed layer transition point regarding defects and will be studied in the future.

Defining the starting point of the extrusion process away from the main contour of the specimens improves the part quality. It avoids an asymmetry of the properties if the selected additive fabricator extrudes too much material at the starting point. The material extruded outside of the part has to be removed by twisting or bending the excess plastic off.

Experimenting with extrusion temperatures of up to $513 \mathrm{~K}$ in $10 \mathrm{~K}$ steps, the optimum temperature for manufacturing compression specimens is $493 \mathrm{~K}$. At lower temperatures, the tracks do not bond. At higher temperatures, gaps appear in the part due to thermal expansion and shrinkage. $503 \mathrm{~K}$ is the optimum extrusion temperature for tensile specimens. The rectangular monolayers tend to detach from the print bed due to thermal dilatation and shrinkage at higher or lower temperatures.

A length-to-width ratio of 6 is best for flat rectangular monolayers. Increasing or decreasing this ratio by one leads to voids, detachment of the parts, or out-of-plane deformations. It is beneficial to have a high width-to-thickness ratio; in the case of study it was 80 . The higher the ratio, the lower the effect of defects in single tracks. The size of surface waves decreases as well, thus increasing the contact area with the grips.

Specimens with parallel trajectories (design 1; see Figure 3) are very hard to manufacture without severe outof-plane deformation due to thermal effects. Creating the geometry starting at the outer contour leads to higher geometrical precision. For this work, the optimum design is a loop-based design with a small theoretical hole in the center (design 5). The void compensates any excess material extrusion. This way, the specimens are flat in the grip area. Tensile specimens design 5 and the selected compression specimen can be seen in Figure 6. They lead to satisfactory micro- and macrogeometrical evaluation results.

The best specimens were manufactured in higher numbers following the findings from the manufacturing experiments. The compression specimens have a diameter-toheight ratio of two due to buckling criteria.

The effect of bed leveling and of the z-gap between print head and print bed cannot be quantified. While the additive fabricator displays a nominal value for the $\mathrm{z}$-gap, this value is not accurate. Measurements with a caliper gauge or other measuring instruments are difficult to realize. Even so, a too high z-gap leads to in-plane and out-of-plane deformation, such as undulated tracks or curved specimens. A too small z-gap causes porosity of the part. This porosity shows itself in two different forms: big gas bubbles that cause the extrusion to fail or mostly dissolved micro gas bubbles that make the part more opaque. The effect of incorrect bed leveling is 


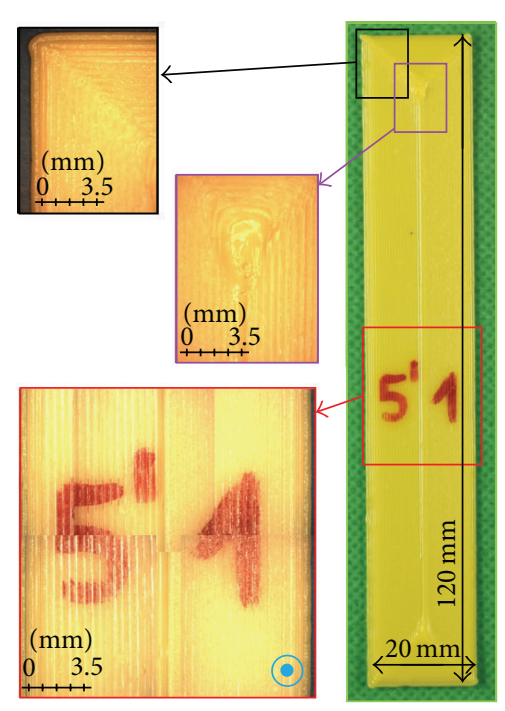

(a)



(b)

FIgURE 6: Typical macrostructure of selected specimens (blue = build direction). (a) Tensile specimen and (b) compression specimen.



FIGURE 7: Break surface of a rectangular monolayer specimen after tensile testing (blue = build direction).

mostly identical to the one caused by an inadequate z-gap. Because of the inclination of the print bed, gas bubbles or deformations may occur in different regions of the extruded part, making the defects' cause more difficult to diagnose in the finished part.

3.2. Mechanical Properties and Behavior. The majority of the tensile specimens with trajectory design 5 fracture outside the grips, validating the use of the file-teeth grips and the grip tightening process. As crazing is well visible on the yellow PLA specimens, the crack formation and propagation can be observed on the video recordings. A break surface analysis shows several internal, hidden gas bubbles that debilitate the material where the failures occurred; see Figure 7 . The figure also shows that the extrusion of marginally more material than necessary in combination with the unheated print bed causes tear-shaped cross sections of the tracks.

As the highest strength value is measured here, the optimum test speed is $2 \mathrm{~mm} / \mathrm{min}$ (equivalent strain rate $0.00042 \mathrm{~s}^{-1}$ ) for determining tensile strength; see Figure 8 . The test speed for determining the corresponding modulus of elasticity is $2 \mathrm{~mm} / \mathrm{min}$ as well.

For compression testing, the optimum test speed is also approximately $2 \mathrm{~mm} / \mathrm{min}$, for both strength and modulus 
TABLE 1: Strength and modulus comparison.

\begin{tabular}{lccccc}
\hline & Specimen type & Test speed [mm/min] & Modulus [MPa] & Strength [MPa] & Elongation at break [\%] \\
\hline \multirow{4}{*}{ Tensile } & Filament & 2 & $2060 \pm 633$ & $51.4 \pm 0.9$ & $17.2 \pm 8.4$ \\
& Monolayer & 2 & $2180 \pm 54$ & $47.0 \pm 0.6$ & $5.1 \pm 2.2$ \\
& PLA [17] & $>12.5$ & 3500 & 53 & 6.0 \\
\hline \multirow{2}{*}{ Compression } & Monolayer & 2 & $1896 \pm 45$ & $58.2 \pm 1.1$ & n.a. \\
& PLA [18] & 0.34 & 2300 & 108 & n.a. \\
\hline
\end{tabular}

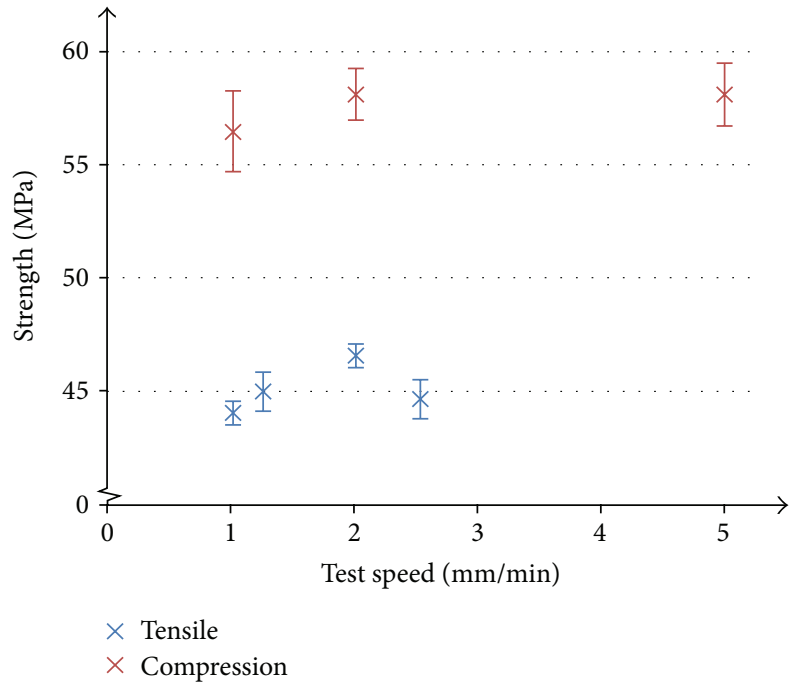

FIGURE 8: AM monolayer tensile and compressive strength at different test speeds.

tests. As the increments between the different test speeds are relatively large, the real optimum test speeds may differ slightly from these values. In future works, further tests may narrow down the possible optimum test speed range.

A test speed of $2 \mathrm{~mm} / \mathrm{min}$ can also be found in ISO 527-5, DIN 65469, and ISO 604 standards that are applicable to plastic and fiber-reinforced plastic materials, respectively. This emphasizes that existing norms may be used as guidelines until new standards are defined for AM. But they may not be applied directly without critical review of the details of their parameter definition and testing procedure. For example, file-teeth grips are necessary for the testing of FLM tensile specimens because the contact area with the grips is small due to the surface undulation. For injection-molded specimens, this is not the case.

The mechanical properties of the monolayers are compared with values for the raw material in Table 1 . The monolayers deviate by $-8.6 \%$ from the tensile strength measured in the reference tests on unextruded filament. Taking into account that the monolayers are composed of fused individual tracks, they have high strength. This confirms that the selected rectangular specimen design is suitable for tensile tests.

The stress whitening (or crazing) that is visible in various locations across the specimens is representative of a fairly uniform stress concentration during the test. The reference tests only differ by $-3.6 \%$ from literature values. While the tensile strength is very similar for the reference test and the monolayers, it seems that the selected PLA type has a lower stiffness than possible for the material.

The compression strength and modulus fall relatively short of the obtainable values when compared with the results of the tensile tests. This might be partly due to the low number of available reference compression tests performed under ISO conditions for PLA. The value used as a comparison base was measured using a higher test speed. Mostly, the difference should be due to still existing optimization potential of the input parameters for monolayer compression specimens. During mechanical testing, some buckling occurred after reaching the compression strength. Also, the fixed layer transition point may locally strengthen the specimens, debilitating them in other areas. The design of these specimens will be improved in future studies.

\section{Conclusions}

Based on the need for standardization in AM, a possible methodology for the parameter definition, manufacturing, quality evaluation, and posttest analysis of monolayer specimens manufactured with FLM was defined. Understanding the behavior of monolayers is vital as they are basic elements of every AM part. Systematically manufacturing and characterizing monolayers was an important step towards this understanding.

In the context of the proposed methodology, a first systematic design, manufacturing via FLM, and testing of monolayer specimens were realized. Different trajectory strategies for flat specimens were defined. For compression testing, circular monolayers in the form of tubes were produced. Their defects were evaluated, creating a catalogue of the frequently occurring defects. The effect of some manufacturing parameters, such as the gap between print head and print bed, trajectory strategy, bed leveling, and temperatures, was assessed.

The mechanical properties of the FLM monolayers manufactured from PLA were determined in tensile and compression tests. The mechanical properties were compared with data from reference tests on unextruded filament and with literature values for PLA. The tendency of FLM dumbbell specimens to fracture outside the gauge length could be reproduced in one out of five cases.

It was demonstrated that under controlled conditions FLM monolayer specimens can be used to conduct mechanical tests. The maximum strength of these monolayer specimens is relatively close to that of specimens of the same material manufactured conventionally. This is especially true for the tensile specimens that were designed. They reach 
tensile strength values that are only $8.6 \%$ lower than the values for reference tests on the raw material.

Future works should be focused on the design of testing procedures and standard specimens for each AM process and material. They should correspond to the basic mechanic loads. Also, developing multifunctional specimens and tests for a group of similar AM processes might be recommendable, studying the similarities in mechanical behavior and material processing for different AM processes, machines. and materials.

A deeper understanding of the behavior and properties of single tracks, monolayers, multilayer parts, and their relationship is needed. Also, the methodology that was proposed in this work may be applied to additively manufactured parts with internal reinforcements or microstructures.

\section{Competing Interests}

The authors declare that they have no competing interests.

\section{Acknowledgments}

This work has received financial support from the Andalusian Government and from the European Union (FEDER/FSE).

\section{References}

[1] Wohler's Associates, Wohlers Report 2015: 3D Printing and Additive Manufacturing State of the Industry Annual Worldwide Progress Report, Wohler's Associates, Fort Collins, Colo, USA, 2015.

[2] M. Hashmi, Ed., Comprehensive Materials Processing, Elsevier, Oxford, UK, 2014.

[3] J. Kranz, D. Herzog, and C. Emmelmann, "Design guidelines for laser additive manufacturing of lightweight structures in TiAl6V4," Journal of Laser Applications, vol. 27, no. 1, Article ID S14001, 2015.

[4] A. Gebhardt, Understanding Additive Manufacturing, Carl Hanser Verlag, Munich, Germany, 2011.

[5] American Society of Testing and Materials ASTM, "Standard Terminology for Additive Manufacturing Technologies," ASTM F2792-12a, 2009.

[6] S.-H. Ahn, M. Montero, D. Odell, S. Roundy, and P. K. Wright, "Anisotropic material properties of fused deposition modeling ABS," Rapid Prototyping Journal, vol. 8, no. 4, pp. 248-257, 2002.

[7] C. S. Lee, S. G. Kim, H. J. Kim, and S. H. Ahn, "Measurement of anisotropic compressive strength of rapid prototyping parts," Journal of Materials Processing Technology, vol. 187-188, pp. 627630, 2007.

[8] Y. Zhu, X. Tian, J. Li, and H. Wang, “The anisotropy of laser melting deposition additive manufacturing Ti-6.5Al-3.5Mo1.5Zr-0.3Si titanium alloy," Materials \& Design, vol. 67, no. 1, pp. 538-542, 2015.

[9] A. Bellini and S. Güçeri, "Mechanical characterization of parts fabricated using fused deposition modeling," Rapid Prototyping Journal, vol. 9, no. 4, pp. 252-264, 2003.

[10] F. Feenstra, "Road map for additive manufacturing 2014 (SASAM project FP7-NMP-2012-CSA-6-319167),” 2015, http://cordis.europa.eu/docs/results/319167/periodicl-sasam-standardisationroadmap-open-febr-2014.pdf.
[11] K. K. Jurrens, "Standards for the rapid prototyping industry," Rapid Prototyping Journal, vol. 5, no. 4, pp. 169-178, 1999.

[12] M. D. Monzón, Z. Ortega, A. Martínez, and F. Ortega, "Standardization in additive manufacturing: activities carried out by international organizations and projects," The International Journal of Advanced Manufacturing Technology, vol. 76, no. 5, pp. 1111-1121, 2014.

[13] American Society for Testing and Materials (ASTM), Committee F42 on Additive Manufacturing Technologies, 2015, http:// www.astm.org/COMMITTEE/F42.htm.

[14] International Organization for Standardization ISO, "ISO/TC 261 Additive manufacturing," 2015, http://www.iso.org/iso/iso_ technical_committee?Commid=629086.

[15] M. Bertoldi, M. A. Yardimci, C. M. Pistor, S. I. GUyeri, and G. Sala, "Mechanical characterization of parts processed via fused deposition," Materials \& Design, vol. 31, no. 1, pp. 287-295, 1998.

[16] J. F. Rodríguez, J. P. Thomas, and J. E. Renaud, "Mechanical behavior of acrylonitrile butadiene styrene (ABS) fused deposition materials. Experimental investigation," Rapid Prototyping Journal, vol. 7, no. 3, pp. 148-158, 2001.

[17] A. Mohanty, M. Misra, and L. Drzal, Eds., Natural Fibers, Biopolymers, and Biocomposites, CRC Press, Boca Raton, Fla, USA, 2005.

[18] T. Yamamura, M. Omiya, T. Sakai, and P. Viot, "Evaluation of compressive properties of PLA/PBAT polymer blends," in Proceedings of the Asian Pacific Conference for Materials and Mechanics, Yokohama, Japan, November 2009.

[19] F. Signori, M.-B. Coltelli, and S. Bronco, "Thermal degradation of poly(lactic acid) (PLA) and poly(butylene adipate-coterephthalate) (PBAT) and their blends upon melt processing," Polymer Degradation and Stability, vol. 94, no. 1, pp. 74-82, 2009.

[20] K. Szykiedans and W. Credo, "Mechanical properties of FDM and SLA low-cost 3-D prints," Procedia Engineering, vol. 136, pp. 257-262, 2016.

[21] L. R. Meza and J. R. Greer, "Mechanical characterization of hollow ceramic nanolattices," Journal of Materials Science, vol. 49, no. 6, pp. 2496-2508, 2014. 

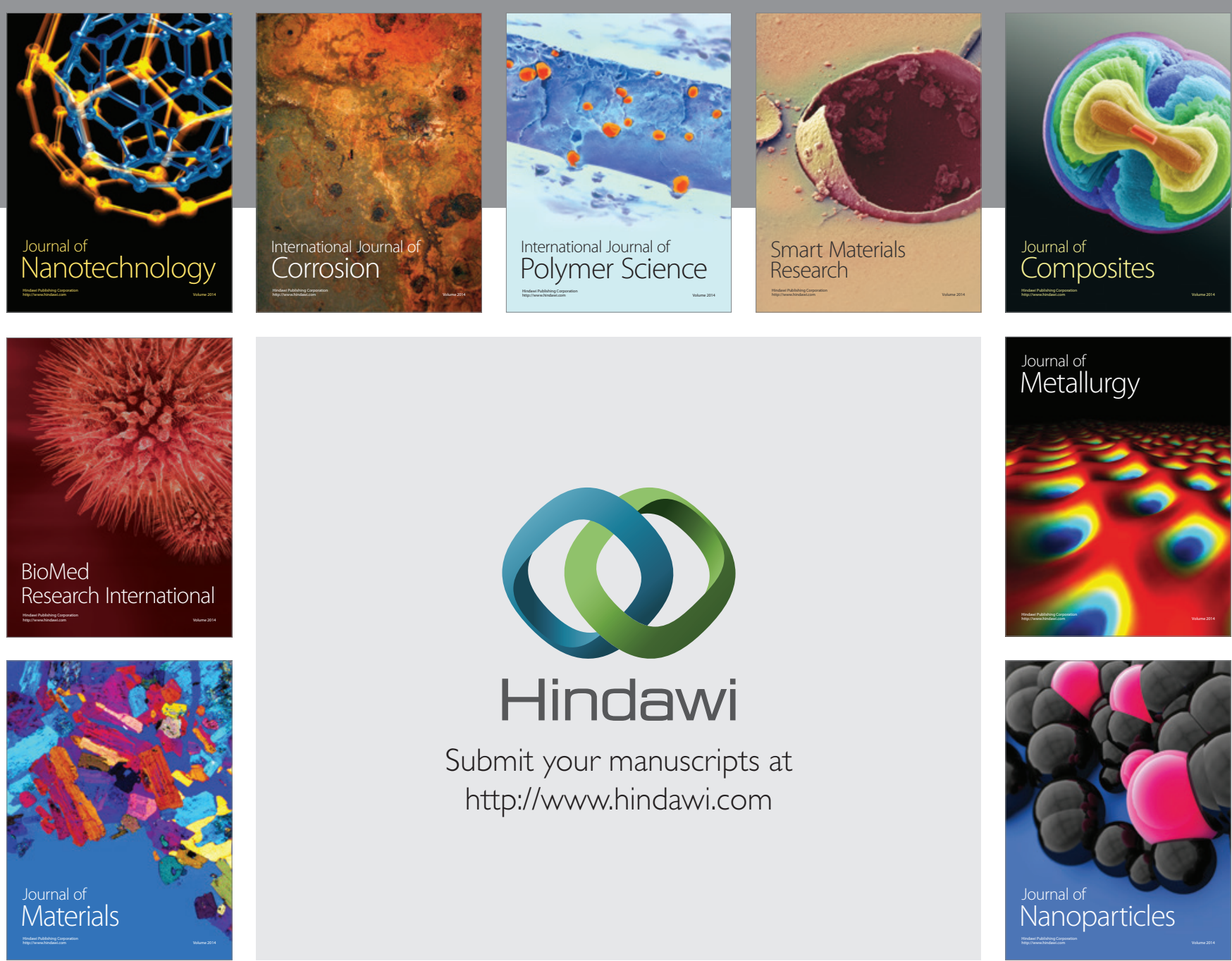

\section{Hindawi}

Submit your manuscripts at

http://www.hindawi.com

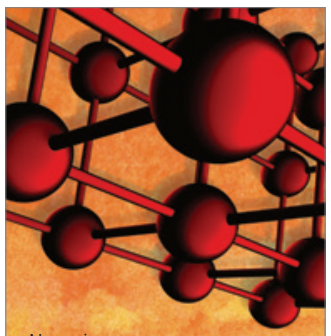

Materials Science and Engineering


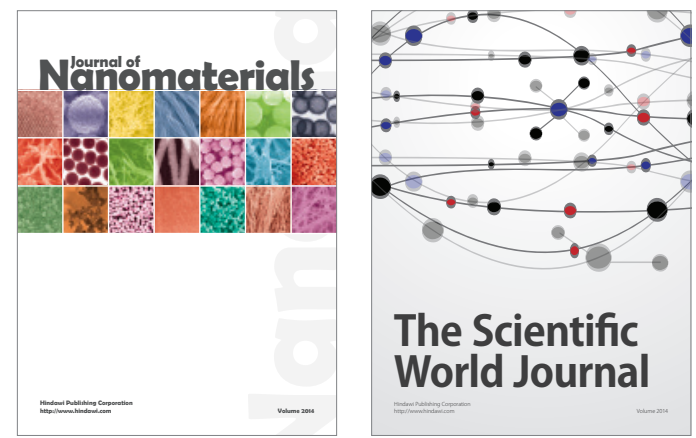

The Scientific World Journal
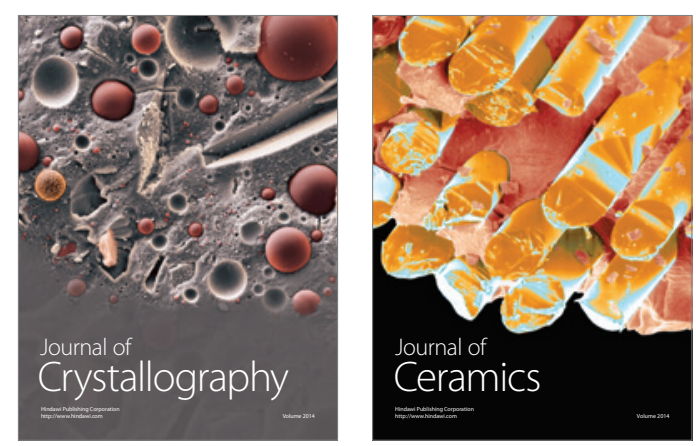
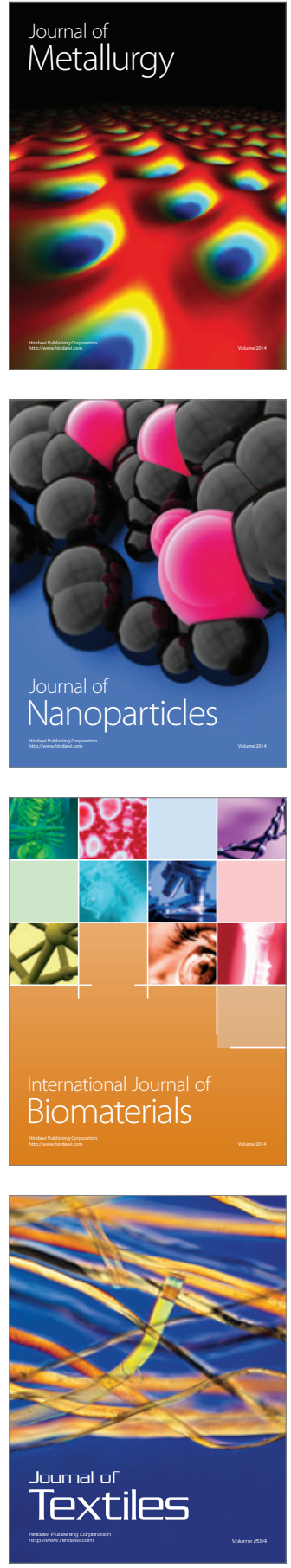